IJMMS 27:4 (2001) 201-214

PII. S0161171201006767

http://ijmms.hindawi.com

(c) Hindawi Publishing Corp.

\title{
MULTIVARIABLE DIMENSION POLYNOMIALS AND NEW INVARIANTS OF DIFFERENTIAL FIELD EXTENSIONS
}

\author{
ALEXANDER B. LEVIN
}

(Received 16 February 2001)

\begin{abstract}
We introduce a special type of reduction in the ring of differential polynomials and develop the appropriate technique of characteristic sets that allows to generalize the classical Kolchin's theorem on differential dimension polynomial and find new differential birational invariants of a finitely generated differential field extension.
\end{abstract}

2000 Mathematics Subject Classification. 12H05, 12H20, 13N15.

1. Introduction. The role of Hilbert polynomials in commutative algebra and algebraic geometry is well known. A similar role in differential algebra is played by differential dimension polynomials. The notion of a differential dimension polynomial was introduced by Kolchin in [6], but the problems and ideas that had led to this concept have essentially more long history. Actually, the differential dimension polynomial describes in exact terms the freedom degree of a dynamic system as well as the number of arbitrary constants in the general solution of a system of algebraic differential equations. The first attempts of such a description were made in the 19th century by Jacobi [3] who estimated the number of algebraically independent constants in the general solution of a system of ordinary linear differential equations. Later on, Jacobi's results were extended to some nonlinear systems, but in the general case the problem of such estimation (known as the problem of Jacobi's bound) remains open.

Differential algebra as a separate area of mathematics is largely due to its founder Ritt (1893-1951) and Kolchin (1916-1991). In 1964 Kolchin proved his famous theorem on differential dimension polynomial (see Theorem 2.1 below) that lies in the foundation of the theory of differential dimension. At the International Congress of Mathematicians in Moscow (1966) Kolchin formulated the main problems and outlined the most perspective directions of research connected with the differential dimension polynomial. Later on the results obtained in this area were included into his famous monograph [7] that hitherto remains the most fundamental work on differential algebra.

Discussing the history of creation of the differential dimension theory, one should note that in 1953 Einstein [2] introduced a concept of strength of a system of differential equations as a certain function of integer argument associated with the system. In 1980 Mikhalëv and Pankrat'ev [12] showed that this function actually coincides with the appropriate differential dimension polynomial and found the strength of some well-known systems of partial differential equations using methods of differential algebra. 
The intensive study of Kolchin's differential dimension polynomials began at the end of the sixties with the series of works by Johnson $[4,5,15]$ who developed the technique of dimension polynomials for differential modules and applied it to the study of some classical problems of differential algebra. In particular, he characterized the Krull dimension of finitely generated differential algebras, developed the theory of local differential algebras, and proved a special case of Janet conjecture. A number of interesting properties and applications of differential dimension polynomials were found by Kondrat'eva, Levin, Mikhalëv, Pankrat'ev, Sit, and some other mathematicians (see $[9,10,11,12,13,14]$ ). One of the most important directions of this study was the search for new differential birational invariants connected with the differential dimension polynomials. Here we should mention the results of Sit [13] who showed that the set of all differential dimension polynomials is well ordered with respect to some natural ordering and introduced the notion of the minimal differential dimension polynomial associated with a differential field extension.

In this paper, we introduce a special type of reduction in a ring of differential polynomials over a differential field of zero characteristic whose basic set is represented as a disjoint union of its subsets. Using the idea of the Gröbner basis method introduced in [1], we develop the appropriate technique of characteristic sets that allows to prove the existence and outline a method of computation of multivariable dimension polynomials associated with a finitely generated differential field extension. In particular, we obtain a generalization of the Kolchin's theorem and find new differential birational invariants.

2. Preliminaries. Throughout the paper $\mathbb{Z}, \mathbb{N}$, and $\mathbb{Q}$ denote the sets of all integers, all nonnegative integers, and all rational numbers, respectively. By a ring we always mean an associative ring with a unit. Every ring homomorphism is unitary (maps unit onto unit), every subring of a ring contains the unit of the ring, and every algebra over a commutative ring is unitary. Unless otherwise indicated, every field is supposed to have zero characteristic.

A differential ring is a commutative ring $R$ considered together with a finite set $\Delta$ of mutually commuting derivations of the ring $R$ into itself. The set $\Delta$ is called a basic set of the differential ring $R$ that is also called a $\Delta$-ring. A subring (ideal) $R_{0}$ of a $\Delta$-ring $R$ is called a differential or $\Delta$-subring of $R$ (respectively, differential or $\Delta$-ideal of $R$ ) if $R_{0}$ is closed with respect to the action of any operator $\delta \in \Delta$. If a differential ( $\Delta$-)ring is a field, it is called a differential (or $\Delta$-)field.

Let $R$ and $S$ be two differential rings with the same basic set $\Delta=\left\{\delta_{1}, \ldots, \delta_{m}\right\}$, so that elements of the set $\Delta$ act on each of the rings as mutually commuting derivations. A ring homomorphism $\phi: R \rightarrow S$ is called a differential or $\Delta$-homomorphism if $\phi(\delta a)=$ $\delta \phi(a)$ for any $\delta \in \Delta, a \in R$.

In what follows, $K$ denotes a differential field whose basic set of derivation operators $\Delta$ is a union of $p$ disjoint finite sets $(p \geq 1): \Delta=\Delta_{1} \cup \cdots \cup \Delta_{p}$, where $\Delta_{i}=$ $\left\{\delta_{i 1}, \ldots, \delta_{i m_{i}}\right\}\left(i=1, \ldots, p\right.$ and $m_{1}, \ldots, m_{p}$ are positive integers whose sum is equal to $m$, the number of elements of the set $\Delta$ ). In other words, we fix a partition of the basic set $\Delta$. Let $\Theta_{i}$ be the free commutative semigroup generated by the elements of the set $\Delta_{i}(i=1, \ldots, p)$ and $\Theta$ the free commutative semigroup generated 
by the whole set $\Delta$. For any element $\theta=\delta_{11}^{k_{11}} \cdots \delta_{1 m_{1}}^{k_{1 m_{1}}} \delta_{21}^{k_{21}} \cdots \delta_{p m_{p}}^{k_{p m_{p}}} \in \Theta$, the numbers $\operatorname{ord}_{i} \theta=\sum_{j=1}^{m_{i}} k_{i j}(i=1, \ldots, p)$ and ord $\theta=\sum_{i=1}^{p} \operatorname{ord}_{i} \theta$ will be called the order of $\theta$ with respect to $\Delta_{i}$ and the order of $\theta$, respectively. As usual, if $\theta, \theta^{\prime} \in \Theta$, we say that $\theta^{\prime}$ divides $\theta$ if $\theta=\theta^{\prime \prime} \theta^{\prime}$ for some element $\theta^{\prime \prime} \in \Theta$. By the least common multiple of the elements $\theta_{1}=\prod_{i=1}^{p} \prod_{j=1}^{m_{i}} \delta_{i j}^{k_{i j 1}}, \ldots, \theta_{q}=\prod_{i=1}^{p} \prod_{j=1}^{m_{i}} \delta_{i j}^{k_{i j q}} \in \Theta$ we mean the element $\theta=\prod_{i=1}^{p} \prod_{j=1}^{m_{i}} \delta_{i j}^{k_{i j}}$, where $k_{i j}=\max \left\{k_{i j l} \mid 1 \leq l \leq q\right\} \quad\left(1 \leq i \leq p, 1 \leq j \leq m_{i}\right)$. This element will be denoted by $\operatorname{lcm}\left(\theta_{1}, \ldots, \theta_{q}\right)$.

For any $r_{1}, \ldots, r_{p}, r \in \mathbb{N}$, the sets $\left\{\theta \in \Theta \mid \operatorname{ord}_{i} \theta \leq r_{i}\right.$ for $\left.i=1, \ldots, p\right\}$ and $\{\theta \in \Theta \mid$ ord $\theta \leq r\}$ will be denoted by $\Theta\left(r_{1}, \ldots, r_{p}\right)$ and $\Theta(r)$, respectively. Furthermore, we set $T \xi=\{\theta(\xi) \mid \theta \in T\}$ for any $\xi \in K, T \subseteq \Theta$.

Below we will consider $p$ orderings $<_{1}, \ldots,<_{p}$ on $\Theta$ defined as follows:

$$
\theta=\delta_{11}^{k_{11}} \cdots \delta_{1 m_{1}}^{k_{1 m_{1}}} \delta_{21}^{k_{21}} \cdots \delta_{p m_{p}}^{k_{p m_{p}}}<_{i} \theta^{\prime}=\delta_{11}^{l_{11}} \cdots \delta_{1 m_{1}}^{l_{1 m_{1}}} \delta_{21}^{l_{21}} \cdots \delta_{p m_{p}}^{l_{p m p}}
$$

if and only if the vector $\left(\operatorname{ord}_{i} \theta, \operatorname{ord} \theta, \operatorname{ord}_{1} \theta, \ldots, \operatorname{ord}_{i-1} \theta, \operatorname{ord}_{i+1} \theta, \ldots, \operatorname{ord}_{p} \theta, k_{i 1}, \ldots\right.$, $\left.k_{i m}, k_{11}, \ldots, k_{1 m_{1}}, k_{21}, \ldots, k_{i-1, m_{i-1}}, k_{i+1,1}, \ldots, k_{p m_{p}}\right)$ is less than the vector $\left(\operatorname{ord}_{i} \theta^{\prime}\right.$, ord $\theta^{\prime}, \operatorname{ord}_{1} \theta^{\prime}, \ldots, \operatorname{ord}_{i-1} \theta^{\prime}, \operatorname{ord}_{i+1} \theta^{\prime}, \ldots, \operatorname{ord}_{p} \theta^{\prime}, l_{i 1}, \ldots, l_{i m}, l_{11}, \ldots, l_{1 m_{1}}, l_{21}, \ldots, l_{i-1, m_{i-1}}$, $l_{i+1,1}, \ldots, l_{p m_{p}}$ ) with respect to the lexicographic order on $\mathbf{N}^{m+p+1}$.

If $R$ is a differential ring with a basic set $\Delta$ and $\Sigma \subseteq R$, then the intersection of all $\Delta$-ideals of $R$ containing the set $\Sigma$ is, obviously, the smallest $\Delta$-ideal of $R$ containing $\Sigma$. This ideal is denoted by [ $\Sigma]$. (It is clear that $[\Sigma]$ is generated, as an ideal, by the set $\{\Theta \xi \mid \xi \in \Sigma\}$.) If the set $\Sigma$ is finite, $\Sigma=\left\{\xi_{1}, \ldots, \xi_{q}\right\}$, we say that the $\Delta$-ideal $I=[\Sigma]$ is finitely generated (we write this as $I=\left[\xi_{1}, \ldots, \xi_{q}\right]$ ) and call $\xi_{1}, \ldots, \xi_{q}$ differential or $\Delta$-generators of $I$.

A subfield $K_{0}$ of the $\Delta$-field $K$ is said to be a differential (or $\Delta$-) subfield of $K$ if $\delta\left(K_{0}\right) \subseteq K_{0}$ for any $\delta \in \Delta$. If $K_{0}$ is a $\Delta$-subfield of the $\Delta$-field $K$ and $\Sigma \subseteq K$, then the intersection of all $\Delta$-subfields of $K$ containing $K_{0}$ and $\Sigma$ is the unique $\Delta$-subfield of $K$ containing $K_{0}$ and $\Sigma$ and contained in every $\Delta$-subfield of $K$ containing $K_{0}$ and $\Sigma$. It is denoted by $K_{0}\langle\Sigma\rangle$. If $K=K_{0}\langle\Sigma\rangle$ and the set $\Sigma$ is finite, $\Sigma=\left\{\eta_{1}, \ldots, \eta_{n}\right\}$, then $K$ is said to be a finitely generated $\Delta$-extension of $K_{0}$ with the set of $\Delta$-generators $\left\{\eta_{1}, \ldots, \eta_{n}\right\}$. In this case we write $K=K_{0}\left\langle\eta_{1}, \ldots, \eta_{n}\right\rangle$. It is easy to see that the field $K_{0}\left\langle\eta_{1}, \ldots, \eta_{n}\right\rangle$ coincides with the field $K_{0}\left(\left\{\theta \eta_{i} \mid \theta \in \Theta, 1 \leq i \leq n\right\}\right)$.

Now we can formulate the Kolchin's theorem on differential dimension polynomial (see [7, Chapter 2, Theorem 6]). As usual, $\left(\begin{array}{l}t \\ k\end{array}\right)(k \in \mathbb{Z}, k \geq 1)$ denotes the polynomial $t(t-1) \cdots(t-k+1) / k$ ! in one variable $t,\left(\begin{array}{l}t \\ 0\end{array}\right)=1$, and $\left(\begin{array}{l}t \\ k\end{array}\right)=0$ if $k<0$.

THEOREM 2.1. Let $K$ be a differential field with a basic set $\Delta=\left\{\delta_{1}, \ldots, \delta_{m}\right\}$ and $L$ a differential field extension of $K$ generated by a finite set $\eta=\left\{\eta_{1}, \ldots, \eta_{n}\right\}$. Then there exists a polynomial $\omega_{\eta \mid K}(t)$ in one variable $t$ with rational coefficients (called $a$ differential dimension polynomial of the extension) such that

(i) $\omega_{\eta \mid K}(r)=\operatorname{trdeg}_{K} K\left(\left\{\theta \eta_{j} \mid \theta \in \Theta(r), 1 \leq j \leq n\right\}\right)$ for all sufficiently large integers $r$;

(ii) $\operatorname{deg} \omega_{\eta \mid K} \leq m$ and the polynomial $\omega_{\eta \mid K}(t)$ can be written as $\omega_{\eta \mid K}(t)=$ $\sum_{i=1}^{m} a_{i}\left(\begin{array}{c}t+i \\ i\end{array}\right)$, where $a_{0}, \ldots, a_{m}$ are some integers; 
(iii) the degree $d$ of the polynomial $\omega_{\eta \mid K}$ and the coefficients $a_{m}$ and $a_{d}$ do not depend on the choice of the system of $\Delta$-generators $\eta$ of the extension $L / K$ (clearly, $a_{d} \neq a_{m}$ if and only if $d<m$, that is, $a_{m}=0$ ). In other words, $d$, $a_{m}$, and $a_{d}$ are differential birational invariants of the extension. Moreover, the coefficient $a_{m}$ is equal to the differential transcendence degree of $L$ over $K$, that is, to the maximal number of elements $\xi_{1}, \ldots, \xi_{k} \in L$ such that the set $\left\{\theta \xi_{i} \mid \theta \in \Theta, 1 \leq i \leq k\right\}$ is algebraically independent over $K$.

If $Y=\left\{y_{1}, \ldots, y_{n}\right\}$ is a finite set of symbols, then one can consider the countable set of symbols $\Theta Y=\left\{\theta y_{j} \mid \theta \in \Theta, 1 \leq j \leq n\right\}$ (called terms) and the polynomial ring $R=$ $K\left[\left\{\theta y_{j} \mid \theta \in \Theta, 1 \leq j \leq n\right\}\right]$ in the set of indeterminates $\Theta Y$ over the differential field $K$. This polynomial ring is naturally viewed as a $\Delta$-ring where $\delta\left(\theta y_{j}\right)=(\delta \theta) y_{j}(\delta \in \Delta$, $\theta \in \Theta, 1 \leq j \leq n)$ and the elements of $\Delta$ act on the coefficients of the polynomials of $R$ as they act in the field $K$. The ring $R$ is called the ring of differential (or $\Delta$-)polynomials in the set of differential $\left(\Delta\right.$-)indeterminates $y_{1}, \ldots, y_{n}$ over the $\Delta$-field $K$. This ring is denoted by $K\left\{y_{1}, \ldots, y_{n}\right\}$ and its elements are called differential (or $\Delta^{-}$) polynomials.

The set of all terms $\Theta Y$ will be considered together with $p$ orderings that correspond to the orderings of the semigroup $\Theta$ and that are denoted by the same symbols $<_{1}, \ldots,<_{p}$. These orderings of $\Theta Y$ are defined as follows: $\theta y_{j}<_{i} \theta^{\prime} y_{k}\left(\theta, \theta^{\prime} \in \Theta, 1 \leq\right.$ $j, k \leq n, 1 \leq i \leq p)$ if and only if $\theta<_{i} \theta^{\prime}$ or $\theta=\theta^{\prime}$ and $j<k$.

By the $i$ th order of a term $u=\theta y_{j}$ we mean the number $\operatorname{ord}_{i} u=\operatorname{ord}_{i} \theta$. The number ord $u=\operatorname{ord} \theta$ is called the order of the term $u$.

We say that a term $u=\theta y_{i}$ is divisible by a term $v=\theta^{\prime} y_{j}$ (or $u$ is a multiple of $v$ ) and write $v \mid u$, if $i=j$ and $\theta^{\prime} \mid \theta$. For any terms $u_{1}=\theta_{1} y_{j}, \ldots, u_{q}=\theta_{q} y_{j}$ containing the same $\Delta$-indeterminate $y_{j}(1 \leq j \leq n)$, the term $\operatorname{lcm}\left(\theta_{1}, \ldots, \theta_{q}\right) y_{j}$ is called the least common multiple of $u_{1}, \ldots, u_{q}$, it is denoted by $\operatorname{lcm}\left(u_{1}, \ldots, u_{q}\right)$.

If $A \in K\left\{y_{1}, \ldots, y_{n}\right\}, A \notin K$, and $1 \leq i \leq p$, then the highest with respect to the ordering $<_{i}$ term that appears in $A$ is called the $i$-leader of the $\Delta$-polynomial $A$. It is denoted by $u_{A}^{(i)}$. If $A$ is written as a polynomial in one variable $u_{A}^{(1)}, A=I_{d}\left(u_{A}^{(1)}\right)^{d}+$ $I_{d-1}\left(u_{A}^{(1)}\right)^{d-1}+\cdots+I_{0}$ (the $\Delta$-polynomials $I_{d}, I_{d-1}, \ldots, I_{0}$ do not contain $\left.u_{A}^{(1)}\right)$, then $I_{d}$ is called the leading coefficient of the $\Delta$-polynomial $A$ and the partial derivative $\partial A / \partial u_{A}^{(1)}=d I_{d}\left(u_{A}^{(1)}\right)^{d-1}+(d-1) I_{d-1}\left(u_{A}^{(1)}\right)^{d-2}+\cdots+I_{1}$ is called the separant of $A$. The leading coefficient and the separant of a $\Delta$-polynomial $A$ are denoted by $I_{A}$ and $S_{A}$, respectively.

DEFINITION 2.2. Let $A$ and $B$ be two $\Delta$-polynomials from $K\left\{y_{1}, \ldots, y_{n}\right\}$. We say that $A$ has a lower rank than $B$ and write $r k A<r k B$ if either $A \in K, B \notin K$, or the vector $\left(u_{A}^{(1)}, \operatorname{deg}_{u_{A}^{(1)}} A, \operatorname{ord}_{2} u_{A}^{(2)}, \ldots, \operatorname{ord}_{p} u_{A}^{(p)}\right)$ is less than the vector $\left(u_{B}^{(1)}, \operatorname{deg}_{u_{B}^{(1)}} B, \operatorname{ord}_{2} u_{B}^{(2)}\right.$, $\ldots, \operatorname{ord}_{p} u_{B}^{(p)}$ ) with respect to the lexicographic order (where $u_{A}^{(1)}$ and $u_{B}^{(1)}$ are compared with respect to $<_{1}$ and all other coordinates of the vectors are compared with respect to the natural order on $\mathbb{N}$ ). If the two vectors are equal (or $A \in K$ and $B \in K$ ) we say that the $\Delta$-polynomials $A$ and $B$ are of the same rank and write $r k A=r k B$.

Let $K$ be a $\Delta$-field and $G=K\left\langle\eta_{1}, \ldots, \eta_{n}\right\rangle$ a finitely generated $\Delta$-extension of $K$ with a set of generators $\eta=\left\{\eta_{1}, \ldots, \eta_{n}\right\}$. Then there exists a natural $\Delta$-homomorphism $\Phi_{\eta}$ from the ring of $\Delta$-polynomials $K\left\{y_{1}, \ldots, y_{n}\right\}$ to $G$ such that $\Phi_{\eta}(a)=a$ for any 
$a \in K$ and $\Phi_{\eta}\left(y_{j}\right)=\eta_{j}$ for $j=1, \ldots, n$. If $A \in K\left\{y_{1}, \ldots, y_{n}\right\}$, then the element $\Phi_{\eta}(A)$ is called the value of the $\Delta$-polynomial $A$ at $\eta$, it is denoted by $A(\eta)$. Obviously, the kernel $P$ of the mapping $\Phi_{\eta}$ is a prime $\Delta$-ideal of the ring $K\left\{y_{1}, \ldots, y_{n}\right\}$. This ideal is called the defining ideal of $\eta$ over $K$ or the defining ideal of the $\Delta$-field extension $G=K\left\langle\eta_{1}, \ldots, \eta_{n}\right\rangle$. It is easy to see that if the quotient field $Q$ of the factor $\operatorname{ring} \bar{R}=$ $K\left\{y_{1}, \ldots, y_{n}\right\} / P$ is considered as a $\Delta$-field (where $\delta(u / v)=(v \delta(u)-u \delta(v)) / v^{2}$ for any $u, v \in \bar{R}$ ), then $Q$ is naturally $\Delta$-isomorphic to the field $G$. (The appropriate $\Delta$ isomorphism is identical on $K$ and maps the images of the $\Delta$-indeterminates $y_{1}, \ldots, y_{n}$ in the factor ring $\bar{R}$ onto the elements $\eta_{1}, \ldots, \eta_{n}$, respectively.)

3. Numerical polynomials. A polynomial $f\left(t_{1}, \ldots, t_{p}\right)$ in $p$ variables $t_{1}, \ldots, t_{p}(p \in \mathbb{N}$, $p \geq 1$ ) with rational coefficients is called numerical if $f\left(t_{1}, \ldots, t_{p}\right) \in \mathbb{Z}$ for all sufficiently large $\left(t_{1}, \ldots, t_{p}\right) \in \mathbb{Z}^{p}$, that is, there exist $s_{1}, \ldots, s_{p} \in \mathbb{Z}$ such that $f\left(r_{1}, \ldots, r_{p}\right) \in \mathbb{Z}$ as soon as $\left(r_{1}, \ldots, r_{p}\right) \in \mathbb{Z}^{p}$ and $r_{i} \geq s_{i}$ for all $i=1, \ldots, p$.

It is clear that any polynomial with integer coefficients is numerical. As an example of a numerical polynomial with noninteger coefficients one can consider a polynomial of the form $\prod_{i=1}^{p}\left(\begin{array}{c}t_{i} \\ m_{i}\end{array}\right)$ where $m_{1}, \ldots, m_{p} \in \mathbb{N}(p \in \mathbb{N}, p \geq 1)$.

If $f\left(t_{1}, \ldots, t_{p}\right)$ is a numerical polynomial, then $\operatorname{deg} f$ and $\operatorname{deg}_{t_{i}} f(1 \leq i \leq p)$ will denote the total degree of $f$ and the degree of $f$ relative to the variable $t_{i}$, respectively. The following theorem proved in [8] gives the "canonical" representation of a numerical polynomial in several variables.

THEOREM 3.1. Let $f\left(t_{1}, \ldots, t_{p}\right)$ be a numerical polynomial in $p$ variables $t_{1}, \ldots, t_{p}$, and let $\operatorname{deg}_{t_{i}} f=m_{i}\left(m_{1}, \ldots, m_{p} \in \mathbb{N}\right)$. Then the polynomial $f\left(t_{1}, \ldots, t_{p}\right)$ can be represented in the form

$$
f\left(t_{1}, \ldots, t_{p}\right)=\sum_{i_{1}=0}^{m_{1}} \cdots \sum_{i_{p}=0}^{m_{p}} a_{i_{1} \cdots i_{p}}\left(\begin{array}{c}
t_{1}+i_{1} \\
i_{1}
\end{array}\right) \cdots\left(\begin{array}{c}
t_{p}+i_{p} \\
i_{p}
\end{array}\right)
$$

with integer coefficients $a_{i_{1} \cdots i_{p}}\left(0 \leq i_{k} \leq m_{k}\right.$ for $\left.k=1, \ldots, p\right)$ that are uniquely defined by the numerical polynomial.

In the rest of the section we deal with subsets of $\mathbb{N}^{m}$ where the positive integer $m$ is represented as a sum of $p$ nonnegative integers $m_{1}, \ldots, m_{p}(p \in \mathbb{N}, p \geq 1)$. In other words, we fix a partition $\left(m_{1}, \ldots, m_{p}\right)$ of the number $m$.

If $\mathscr{A} \subseteq \mathbb{N}^{m}$ and $r_{1}, \ldots, r_{p} \in \mathbb{N}$, then $\mathscr{A}\left(r_{1}, \ldots, r_{p}\right)$ will denote the set $\left\{\left(a_{1}, \ldots, a_{m}\right) \in\right.$ $\left.\mathscr{A} \mid a_{1}+\cdots+a_{m_{1}} \leq r_{1}, a_{m_{1}+1}+\cdots+a_{m_{1}+m_{2}} \leq r_{2}, \ldots, a_{m_{1}+\cdots+m_{p-1}+1}+\cdots+a_{m} \leq r_{p}\right\}$. Furthermore, $V_{\mathscr{A}}$ will denote the set of all $m$-tuples $v=\left(v_{1}, \ldots, v_{m}\right) \in \mathbb{N}^{m}$ that are not greater than or equal to any $m$-tuple from $\mathscr{A}$ with respect to the product order on $\mathbb{N}^{m}$. (Recall that the product order on the set $\mathbb{N}^{k}(k \in \mathbb{N}, k \geq 1)$ is a partial order $\leq_{P}$ such that $c=\left(c_{1}, \ldots, c_{k}\right) \leq_{P} c^{\prime}=\left(c_{1}^{\prime}, \ldots, c_{k}^{\prime}\right)$ if and only if $c_{i} \leq c_{i}^{\prime}$ for all $i=1, \ldots, k$. If $c \leq_{P} c^{\prime}$ and $c \neq c^{\prime}$, we write $\left.c<_{P} c^{\prime}\right)$. Clearly, an element $v=\left(v_{1}, \ldots, v_{m}\right) \in \mathbb{N}^{m}$ belongs to $V_{\mathscr{A}}$ if and only if for any $\left(a_{1}, \ldots, a_{m}\right) \in \mathscr{A}$, there exists $i \in \mathbb{N}, 1 \leq i \leq m$, such that $a_{i}>v_{i}$.

The following two theorems proved in [8] generalize the well-known Kolchin's result on the numerical polynomials associated with subsets of $\mathbb{N}$ (see [7, Chapter 0 , Lemma 17]) and give the explicit formula for the numerical polynomials in $p$ variables associated with a finite subset of $\mathbb{N}^{m}$. 
THEOREM 3.2. Let $A$ be a subset of $\mathbb{N}^{m}$ where $m=m_{1}+\cdots+m_{p}$ for some nonnegative integers $m_{1}, \ldots, m_{p}(p \geq 1)$. Then there exists a numerical polynomial $\omega_{\mathscr{A}}\left(t_{1}, \ldots, t_{p}\right)$ with the following properties:

(i) $\omega_{\mathscr{A}}\left(r_{1}, \ldots, r_{p}\right)=$ Card $V_{\mathscr{A}}\left(r_{1}, \ldots, r_{p}\right)$ for all sufficiently large $\left(r_{1}, \ldots, r_{p}\right) \in \mathbb{N}^{p}$ (as usual, $C \operatorname{ard} M$ denotes the number of elements of a finite set $M$ ).

(ii) $\operatorname{deg} \omega_{\mathscr{A}} \leq m$ and $\operatorname{deg}_{t_{i}} \omega_{\mathscr{A}} \leq m_{i}$ for $i=1, \ldots, p$.

(iii) $\operatorname{deg} \omega_{\mathscr{A}}=m$ if and only if $\mathscr{A}=\varnothing$. In this case, $\omega_{\mathscr{A}}\left(t_{1}, \ldots, t_{p}\right)=\prod_{i=1}^{p}\left(\begin{array}{c}t_{i}+m_{i} \\ m_{i}\end{array}\right)$.

(iv) $\omega_{\mathscr{A}}$ is a zero polynomial if and only if $(0, \ldots, 0) \in A$.

The polynomial $\omega_{\mathscr{A}}\left(t_{1}, \ldots, t_{p}\right)$ whose existence is stated by Theorem 3.2 is called the dimension polynomial of the set $\mathscr{A} \subseteq \mathbb{N}^{m}$ associated with the partition $\left(m_{1}, \ldots, m_{p}\right)$ of $m$. If $p=1$, the polynomial $\omega_{\mathscr{A}}$ is called the Kolchin polynomial of the set $\mathscr{A}$.

THEOREM 3.3. Let $\mathscr{A}=\left\{a_{1}, \ldots, a_{n}\right\}$ be a finite subset of $\mathbb{N}^{m}$ and $\left(m_{1}, \ldots, m_{p}\right)(p \geq 1)$ a partition of $m$. Let $a_{i}=\left(a_{i 1}, \ldots, a_{i m}\right)(1 \leq i \leq n)$ and for any $l \in \mathbb{N}, 0 \leq l \leq n$, let $\Gamma(l, n)$ denote the set of all l-element subsets of the set $\mathbb{N}_{n}=\{1, \ldots, n\}$. Furthermore, for any $\sigma \in \Gamma(l, p)$, let $\bar{a}_{\sigma j}=\max \left\{a_{i j} \mid i \in \sigma\right\}(1 \leq j \leq m)$ and $b_{\sigma j}=\sum_{h \in \sigma j} \bar{a}_{\sigma h}$. Then

$$
\omega_{\mathscr{A}}\left(t_{1}, \ldots, t_{p}\right)=\sum_{l=0}^{n}(-1)^{l} \sum_{\sigma \in \Gamma(l, p)} \prod_{j=1}^{n}\left(\begin{array}{c}
t_{j}+m_{j}-b_{\sigma j} \\
m_{j}
\end{array}\right) .
$$

It is clear that if $\mathscr{A} \subseteq \mathbb{N}^{m}$ and $\mathscr{A}^{\prime}$ is the set of all minimal elements of the set $\mathscr{A}$ with respect to the product order on $\mathbb{N}^{m}$, then the set $\mathscr{A}^{\prime}$ is finite and $\omega_{\mathscr{A}}\left(t_{1}, \ldots, t_{p}\right)=$ $\omega_{\mathscr{A} \mathfrak{A}^{\prime}}\left(t_{1}, \ldots, t_{p}\right)$. Thus, Theorem 3.3 gives an algorithm that allows to find a numerical polynomial associated with any subset of $\mathbb{N}^{m}$ (and with a given partition of $m$ ): one should first find the set of all minimal points of the subset and then apply Theorem 3.3.

4. Reduction in the ring of differential polynomials. In what follows we keep the notation and conventions of Section 2. In particular, $K\left\{y_{1}, \ldots, y_{n}\right\}$ denotes the ring of $\Delta$-polynomials over a differential field $K$ whose basic set $\Delta$ is a union of $p$ disjoint sets: $\Delta=\Delta_{1} \cup \cdots \cup \Delta_{p}$, where $\Delta_{i}=\left\{\delta_{i 1}, \ldots, \delta_{i m_{i}}\right\}(1 \leq i \leq p)$.

Definition 4.1. Let $A, B \in K\left\{y_{1}, \ldots, y_{n}\right\}$ and $A \notin K$. The $\Delta$-polynomial $B$ is said to be reduced with respect to $A$ if the following two conditions hold:

(i) $B$ does not contain any term $\theta u_{A}^{(1)}(\theta \in \Theta, \theta \neq 1)$ such that $\operatorname{ord}_{i}\left(\theta u_{A}^{(i)}\right) \leq \operatorname{ord}_{i} u_{B}^{(i)}$ for $i=2, \ldots, p$.

(ii) If $B$ contains $u_{A}^{(1)}$, then either $\operatorname{ord}_{j} u_{B}^{(j)}<\operatorname{ord}_{j} u_{A}^{(j)}$ for some $j(2 \leq j \leq p)$ or $\operatorname{ord}_{i} u_{A}^{(i)} \leq \operatorname{ord}_{i} u_{B}^{(i)}$ for all $i=2, \ldots, p$ and $\operatorname{deg}_{u_{A}^{(1)}} B<\operatorname{deg}_{u_{A}^{(1)}} A$.

A $\Delta$-polynomial $B$ is said to be reduced with respect to a set of $\Delta$-polynomials $\Sigma \subseteq K\left\{y_{1}, \ldots, y_{n}\right\}$ if $B$ is reduced with respect to every element of $\Sigma$.

DEFINITION 4.2. A set of $\Delta$-polynomials $\Sigma \subseteq K\left\{y_{1}, \ldots, y_{n}\right\}$ is called autoreduced if $\Sigma \bigcap K=\varnothing$ and every element of $\Sigma$ is reduced with respect to any other element of this set.

The proof of the following lemma can be found in [7, Chapter 0, Section 17]. 
LEMmA 4.3. Let $\mathbb{N}_{n}=\{1, \ldots, n\}$ and let $A$ be an infinite subset of $\mathbb{N}^{m} \times \mathbb{N}_{n}(m, n \in \mathbb{N}$, $n \geq 1$ ). Then there exists an infinite sequence of elements of $A$, strictly increasing relative to the product order, in which every element has the same projection on $\mathbb{N}_{n}$.

This result implies the following statement that will be used below.

LEMMA 4.4. Let $S$ be an infinite set of terms in the ring $K\left\{y_{1}, \ldots, y_{n}\right\}$. Then there exists an index $j(1 \leq j \leq n)$ and an infinite sequence of terms $\theta_{1} y_{j}, \theta_{2} y_{j}, \ldots, \theta_{k} y_{j}, \ldots \in S$ such that $\theta_{k} \mid \theta_{k+1}$ for all $k=1,2, \ldots$.

THEOREM 4.5. Every autoreduced set of $\Delta$-polynomials is finite.

Proof. Suppose that $\Sigma$ is an infinite autoreduced subset of $K\left\{y_{1}, \ldots, y_{n}\right\}$. Then $\Sigma$ contains an infinite set $\Sigma^{\prime}$ such that all $\Delta$-polynomials from $\Sigma^{\prime}$ have different 1leaders. Indeed, if it is not so, then there exists an infinite set $\Sigma_{1} \subseteq \Sigma$ such that all $\Delta$-polynomials from $\Sigma_{1}$ have the same 1-leader $u$. By Lemma 4.3, the infinite set $\left\{\left(\operatorname{ord}_{2} u_{A}^{(2)}, \ldots, \operatorname{ord}_{p} u_{A}^{(p)}\right) \mid A \in \Sigma_{1}\right\}$ contains a nondecreasing infinite sequence $\left(\operatorname{ord}_{2} u_{A_{1}}^{(2)}, \ldots, \operatorname{ord}_{p} u_{A_{1}}^{(p)}\right) \leq_{P}\left(\operatorname{ord}_{2} u_{A_{2}}^{(2)}, \ldots, \operatorname{ord}_{p} u_{A_{2}}^{(p)}\right) \leq_{P} \cdots\left(A_{1}, A_{2}, \ldots \in \Sigma_{1}\right.$ and $\leq_{P}$ denotes the product order on $\left.\mathbb{N}^{p-1}\right)$. Since the sequence $\left\{\operatorname{deg}_{u} A_{i} \mid i=1,2, \ldots\right\}$ cannot strictly decrease, there exists two indices $i$ and $j$ such that $i<j$ and $\operatorname{deg}_{u} A_{i} \leq \operatorname{deg}_{u} A_{j}$. We obtain that $A_{j}$ is reduced with respect to $A_{i}$ that contradicts the fact that $\Sigma$ is an autoreduced set.

Thus, we can assume that elements of $\Sigma$ have distinct 1-leaders. By Lemma 4.4, there exists an infinite sequence $B_{1}, B_{2}, \ldots$ of elements of $\Sigma$ such that $u_{B_{i}}^{(1)} \mid u_{B_{i+1}}^{(1)}$ for $i=1,2, \ldots$. Let $k_{i j}=\operatorname{ord}_{j} u_{B_{i}}^{(1)}$ and $l_{i j}=\operatorname{ord}_{j} u_{B_{i}}^{(j)}(2 \leq j \leq p)$. Obviously, $l_{i j} \geq k_{i j}(i=$ $1,2, \ldots ; j=2, \ldots, p)$, so that $\left\{\left(l_{i 2}-k_{i 2}, \ldots, l_{i p}-k_{i p}\right) \mid i=1,2, \ldots\right\} \subseteq \mathbb{N}^{p-1}$. By Lemma 4.3, there exists an infinite sequence of indices $i_{1}<i_{2}<\cdots$ such that $\left(l_{i_{1} 2}-k_{i_{1} 2}, \ldots, l_{i_{1} p}-\right.$ $\left.k_{i_{1} p}\right) \leq_{P}\left(l_{i_{2} 2}-k_{i_{2} 2}, \ldots, l_{i_{2} p}-k_{i_{2} p}\right) \leq_{P} \ldots$. Then for any $j=2, \ldots, p$, we have

$$
\operatorname{ord}_{j}\left(\frac{u_{B_{i_{2}}}^{(1)}}{u_{B_{i_{1}}}^{(1)}} u_{B_{i_{1}}}^{(j)}\right)=k_{i_{2} j}-k_{i_{1} j}+l_{i_{1} j} \leq k_{i_{2} j}+l_{i_{2} j}-k_{i_{2} j}=l_{i_{2} j}=\operatorname{ord}_{j} u_{B_{i_{2}}}^{(j)},
$$

so that $B_{i_{2}}$ contains a term $\theta u_{B_{i_{1}}}^{(1)}=u_{B_{i_{2}}}^{(1)}$ such that $\theta \neq 1$ and $\operatorname{ord}_{j}\left(\theta u_{B_{i_{1}}}^{(j)}\right) \leq \operatorname{ord}_{j} u_{B_{i_{2}}}^{(j)}$ for $j=2, \ldots, p$. Thus, the $\Delta$-polynomial $B_{i_{2}}$ is reduced with respect to $B_{i_{1}}$ that contradicts the fact that $\Sigma$ is an autoreduced set. This completes the proof of the theorem.

Below, the elements of an autoreduced set will be always arranged in order of increasing rank. (Therefore, if we consider an autoreduced set of $\Delta$-polynomials $\Sigma=$ $\left\{A_{1}, \ldots, A_{r}\right\}$, then $r k A_{1}<\cdots<r k A_{r}$.

The following two theorems can be proven precisely in the same way as their classical analogs (see [7, Chapter 1, Corollary to Lemma 6 and Proposition 3, page 78]).

THEOREM 4.6. Let $\Sigma=\left\{A_{1}, \ldots, A_{r}\right\}$ be an autoreduced set in the ring $K\left\{y_{1}, \ldots, y_{n}\right\}$ and let $B$ be a $\Delta$-polynomial. Then there exist a $\Delta$-polynomial $B_{0}$ and nonnegative integers $p_{i}, q_{i}(1 \leq i \leq r)$ such that $B_{0}$ is reduced with respect to $\Sigma, r k B_{0} \leq r k B$, and $\prod_{i=1}^{r} I_{A_{i}}^{p_{i}} S_{A_{i}}^{q_{i}} B \equiv B_{0}(\bmod [\Sigma])$. 
DEFINITION 4.7. Let $\Sigma=\left\{A_{1}, \ldots, A_{r}\right\}$ and $\Sigma^{\prime}=\left\{B_{1}, \ldots, B_{s}\right\}$ be two autoreduced sets in the ring of differential polynomials $K\left\{y_{1}, \ldots, y_{n}\right\}$. An autoreduced set $\Sigma$ is said to have lower rank than $\Sigma^{\prime}$ if one of the following two cases holds:

(1) there exists $k \in \mathbb{N}$ such that $k \leq \min \{r, s\}, r k A_{i}=r k B_{i}$ for $i=1, \ldots, k-1$ and $r k A_{k}<r k B_{k}$

(2) $r>s$ and $r k A_{i}=r k B_{i}$ for $i=1, \ldots, s$.

If $r=s$ and $r k A_{i}=r k B_{i}$ for $i=1, \ldots, r$, then $\Sigma$ is said to have the same rank as $\Sigma^{\prime}$.

THEOREM 4.8. In every nonempty family of autoreduced sets of differential polynomials there exists an autoreduced set of lowest rank.

Let $J$ be an ideal of the ring $K\left\{y_{1}, \ldots, y_{n}\right\}$. Since the family of all autoreduced subsets of $J$ is not empty (e.g., it contains the empty set), Theorem 4.8 shows that the ideal $J$ contains an autoreduced subset of lowest rank.

DEFINITION 4.9. Let $J$ be an ideal of the ring of differential polynomials $K\left\{y_{1}, \ldots\right.$, $\left.y_{n}\right\}$. Then an autoreduced subset of $J$ of lowest rank is called a characteristic set of the ideal $J$.

THEOREM 4.10. Let $\Sigma=\left\{A_{1}, \ldots, A_{d}\right\}$ be a characteristic set of an ideal $J$ of the ring of $\Delta$-polynomials $R=K\left\{y_{1}, \ldots, y_{n}\right\}$. Then an element $B \in R$ is reduced with respect to the set $\Sigma$ if and only if $B=0$.

Proof. Suppose that $B \neq 0$. Then $B$ and elements of $\Sigma$ whose rank is lower than the rank of $B$ form an autoreduced set $\Sigma^{\prime}$. It is easy to see that $\Sigma^{\prime}$ has a lower rank than $\Sigma$ that contradicts the fact that $\Sigma$ is a characteristic set of the ideal $J$.

THEOREM 4.11. Let $J$ be a cyclic differential ideal of the ring of $\Delta$-polynomials $R=$ $K\left\{y_{1}, \ldots, y_{n}\right\}$ generated by a linear $\Delta$-polynomial $f$. Then $\{f\}$ is a characteristic set of the $\Delta$-ideal $J=[f]$.

Proof. First of all, we show that no nonzero element of $J$ is reduced with respect to $f$. Let $0 \neq h \in J$, and let $k$ be the smallest positive integer such that $h$ can be written as

$$
h=g_{1} \theta_{1} f+\cdots+g_{k} \theta_{k} f,
$$

for some pairwise distinct elements $\theta_{1}, \ldots, \theta_{k} \in \Theta$ and some $g_{1}, \ldots, g_{k} \in R$. In what follows, we suppose that $k>1$ (clearly, an element of the form $g \theta f(g \in R, \theta \in \Theta)$ is not reduced with respect to $f$ ) and $\theta_{1}<_{1} \cdots<_{1} \theta_{k}$. Furthermore, it is obvious that $\theta_{k} f \nmid g_{j}$ for $j=1, \ldots, k-1$ (otherwise, $h$ is a linear combination of $k-1$ elements from $\Theta f)$.

Since $\theta_{k} f$ is linear with respect to $\theta_{k} u_{f}^{(1)}$, one can write each $\Delta$-polynomial $g_{j}(1 \leq$ $j \leq k-1)$ as $g_{j}=g_{j}^{\prime}+g_{j}^{\prime \prime}\left(\theta_{k} f\right)$, where $g_{j}^{\prime}, g_{j}^{\prime \prime} \in R$ and $g_{j}^{\prime}$ does not contain $\theta_{k} u_{f}^{(1)}$. Then $h=g_{1}^{\prime} \theta_{1} f+\cdots+g_{k-1}^{\prime} \theta_{k-1} f+g \theta_{k} f$ for some $g \in R$.

Since $g \theta_{k} f$ contains $\theta_{k} u_{f}^{(1)}$ and none of $g_{j}^{\prime} \theta_{j} f(1 \leq j \leq k-1)$ contains this term, the $\Delta$-polynomial $h$ contains $\theta_{k} u_{f}^{(1)}$ and $r k f \leq r k h$. Similarly, if $\theta_{j_{i}}$ is the maximal element of the set $\left\{\theta_{1}, \ldots, \theta_{k}\right\}$ relative to the order $<_{i}$ on $\Theta(2 \leq i \leq p)$, then $h$ contains $\theta_{j_{i}} u_{f}^{(i)}$, so that $\operatorname{ord}_{i}\left(\theta_{k} u_{f}^{(i)}\right) \leq \operatorname{ord}_{i}\left(\theta_{j_{i}} u_{f}^{(i)}\right) \leq \operatorname{ord}_{i} u_{h}^{(i)}$. It follows that $h$ is reduced with 
respect to $f$ and $f$ is an element of the lowest rank in $J$. Therefore, if $\Sigma=\left\{h_{1}, \ldots, h_{l}\right\}$ is a characteristic set of $J$, then $r k f=r k h_{1}$ and $l=1$, whence $\{f\}$ is also a characteristic set of the ideal $J$.

5. Multivariable differential dimension polynomials and their invariants. Now we can prove the main theorem on multivariable differential dimension polynomial that generalizes the classical Kolchin's result (see Theorem 2.1).

THEOREM 5.1. Let $K$ be a differential field whose basic set of derivation operators $\Delta$ is a union of $p$ disjoint finite sets $(p \geq 1): \Delta=\Delta_{1} \cup \cdots \cup \Delta_{p}$, where $\Delta_{i}=\left\{\delta_{i 1}, \ldots, \delta_{i m_{i}}\right\}$ ( $m_{1}, \ldots, m_{p}$ are some positive integers). Furthermore, let $G=K\left\langle\eta_{1}, \ldots, \eta_{n}\right\rangle$ be a $\Delta$-field extension of $K$ generated by a finite set $\eta=\left\{\eta_{1}, \ldots, \eta_{n}\right\}$. Then there exists a polynomial $\Phi_{\eta}\left(t_{1}, \ldots, t_{p}\right)$ in $p$ variables $t_{1}, \ldots, t_{p}$ with rational coefficients such that

(i) $\Phi_{\eta}\left(r_{1}, \ldots, r_{p}\right)=\operatorname{trdeg}_{K} K\left(\bigcup_{j=1}^{n} \Theta\left(r_{1}, \ldots, r_{p}\right) \eta_{j}\right)$ for all sufficiently large $\left(r_{1}, \ldots\right.$, $\left.r_{p}\right) \in \mathbb{Z}^{p}$

(ii) $\operatorname{deg}_{t_{i}} \Phi_{\eta} \leq m_{i}(i=1, \ldots, p)$ and the polynomial $\Phi_{\eta}\left(t_{1}, \ldots, t_{p}\right)$ can be written as

$$
\Phi_{\eta}\left(t_{1}, \ldots, t_{p}\right)=\sum_{i_{1}=0}^{m_{1}} \cdots \sum_{i_{p}=0}^{m_{p}} a_{i_{1} \cdots i_{p}}\left(\begin{array}{c}
t_{1}+i_{1} \\
i_{1}
\end{array}\right) \cdots\left(\begin{array}{c}
t_{p}+i_{p} \\
i_{p}
\end{array}\right),
$$

where $a_{i_{1} \ldots i_{p}} \in \mathbb{Z}$ for all $i_{1}, \ldots, i_{p}$.

Proof. Let $P$ be the defining $\Delta$-ideal of the extension $G / K$ and $\Sigma=\left\{A_{1}, \ldots, A_{d}\right\}$ a characteristic set of $P$. Furthermore, for any $r_{1}, \ldots, r_{p} \in \mathbb{N}$, let $U_{r_{1}} \cdots r_{p}=\{u \in \Theta Y \mid$ $\operatorname{ord}_{i} u \leq r_{i}$ for $i=1, \ldots, p$ and either $u$ is not a multiple of any $u_{A_{i}}^{(1)}$, or for every $\theta \in \Theta$, $A \in \Sigma$ such that $u=\theta u_{A}^{(1)}$, there exists $i \in\{2, \ldots, p\}$ such that $\left.\operatorname{ord}_{i}\left(\theta u_{A}^{(i)}\right)>r_{i}\right\}$. (If $p=1$, we set $U_{r_{1}}=\left\{u \in \Theta Y \mid \operatorname{ord}_{1} u \leq r_{1}\right.$ and $u$ is not a multiple of any $\left.u_{A_{i}}^{(1)}\right\}$.) We are going to show that the set $\bar{U}_{r_{1} \cdots r_{p}}=\left\{u(\eta) \mid u \in U_{r_{1} \cdots r_{p}}\right\}$ is a transcendence basis of the field $K\left(\bigcup_{j=1}^{n} \Theta\left(r_{1}, \ldots, r_{p}\right) \eta_{j}\right)$ over $K$.

First of all, we show that the set $\bar{U}_{r_{1} \ldots r_{p}}$ is algebraically independent over $K$. Let $g$ be a polynomial in $k$ variables $(k \in \mathbb{N}, k \geq 1)$ such that $g\left(u_{1}(\eta), \ldots, u_{k}(\eta)\right)=0$ for some elements $u_{1}, \ldots, u_{k} \in U_{r_{1} \cdots r_{p}}$. Then the $\Delta$-polynomial $\bar{g}=g\left(u_{1}, \ldots, u_{k}\right)$ is reduced with respect to $\Sigma$ and $\bar{g} \in P$. Applying Theorem 4.10 we obtain that $\bar{g}=0$. Thus, the set $\bar{U}_{r_{1} \cdots r_{p}}$ is algebraically independent over $K$.

Now, we show that every element $\theta \eta_{j}\left(1 \leq j \leq n, \theta \in \Theta\left(r_{1}, \ldots, r_{p}\right)\right)$ is algebraic over the field $K\left(\bar{U}_{r_{1}, \ldots, r_{p}}\right)$. Let $\theta \eta_{j} \notin \bar{U}_{r_{1}, \ldots, r_{p}}$ (if $\theta \eta_{j} \in \bar{U}_{r_{1}, \ldots, r_{p}}$, the statement is obvious). Then $\theta y_{j} \notin U_{r_{1}, \ldots, r_{p}}$ whence $\theta y_{j}$ is equal to some term of the form $\theta^{\prime} u_{A_{i}}^{(1)}\left(\theta^{\prime} \in \Theta\right.$, $1 \leq i \leq d)$ such that $\operatorname{ord}_{k}\left(\theta^{\prime} u_{A_{i}}^{(k)}\right) \leq r_{k}$ for $k=2, \ldots, p$. We represent $A_{i}$ as a polynomial in $u_{A_{i}}^{(1)}: A_{i}=I_{0}\left(u_{A_{i}}^{(1)}\right)^{e}+I_{1}\left(u_{A_{i}}^{(1)}\right)^{e-1}+\cdots+I_{e}$, where $I_{0}, I_{1}, \ldots, I_{e}$ do not contain $u_{A_{i}}^{(1)}$ (therefore, all terms in these $\Delta$-polynomials are lower than $u_{A_{i}}^{(1)}$ with respect to the order $<_{1}$ ). Since $A_{i} \in P$,

$$
A_{i}(\eta)=I_{0}(\eta)\left(u_{A_{i}}^{(1)}(\eta)\right)^{e}+I_{1}(\eta)\left(u_{A_{i}}^{(1)}(\eta)\right)^{e-1}+\cdots+I_{e}(\eta)=0
$$

It is clear that $I_{0}$ and $S_{A_{i}}=\partial A_{i} / \partial u_{A_{i}}^{(1)}$ are reduced with respect to the set $\Sigma$. By 
Theorem 4.10, $I_{0} \notin P$ and $S_{A_{i}} \notin P$ whence $I_{0}(\eta) \neq 0$ and $S_{A_{i}}(\eta) \neq 0$. If we apply the operator $\theta^{\prime}$ to both sides of (5.2), the resulted equation will show that the element $\theta^{\prime} u_{A_{i}}^{(1)}(\eta)=\theta \eta_{j}$ is algebraic over the field $K\left(\left\{\bar{\theta} \eta_{l} \mid \operatorname{ord}_{i} \bar{\theta} \leq r_{i}(1 \leq i \leq p, 1 \leq l \leq n)\right.\right.$ and $\left.\left.\bar{\theta} y_{l}<_{1} \theta^{\prime} u_{A_{i}}^{(1)}=\theta y_{j}\right\}\right)$. Now, the induction on the set $\Theta Y$ ordered by the relation $<_{1}$ completes the proof of the fact that $\bar{U}_{r_{1} \cdots r_{p}}(\eta)$ is a transcendence basis of the field $K\left(\bigcup_{j=1}^{n} \Theta\left(r_{1}, \ldots, r_{p}\right) \eta_{j}\right)$ over $K$.

Let $U_{r_{1} \cdots r_{p}}^{(1)}=\left\{u \in \Theta Y \mid \operatorname{ord}_{i} u \leq r_{i}\right.$ for $i=1, \ldots, p$ and $u \neq \theta u_{A_{j}}^{(1)}$ for any $\theta \in \Theta$; $j=1, \ldots, d\}$ and $U_{r_{1}}^{(2)} \cdots r_{p}=\left\{u \in \Theta Y \mid \operatorname{ord}_{i} u \leq r_{i}\right.$ for $i=1, \ldots, p$ and there exists at least one pair $i, j(1 \leq i \leq p, 1 \leq j \leq d)$ such that $u=\theta u_{A_{j}}^{(1)}$ and $\left.\operatorname{ord}_{i}\left(\theta u_{A_{j}}^{(i)}\right)>r_{i}\right\}$. (If $p=1$, then we set $U_{r_{1} \cdots r_{p}}^{(2)}=\varnothing$.) Clearly, $U_{r_{1} \cdots r_{p}}=U_{r_{1} \cdots r_{p}}^{(1)} \cup U_{r_{1} \cdots r_{p}}^{(2)}$ and $U_{r_{1} \cdots r_{p}}^{(1)} \cap U_{r_{1} \cdots r_{p}}^{(2)}=\varnothing$.

By Theorem 3.2, there exists a numerical polynomial $\omega\left(t_{1}, \ldots, t_{p}\right)$ in $p$ variables $t_{1}, \ldots, t_{p}$ such that $\omega\left(r_{1}, \ldots, r_{p}\right)=$ Card $U_{r_{1} \cdots r_{p}}^{(1)}$ for all sufficiently large $\left(r_{1}, \ldots, r_{p}\right) \in \mathbb{Z}^{p}$ and $\operatorname{deg}_{t_{i}} \omega \leq m_{i}(i=1, \ldots, p)$. Thus, in order to complete the proof of the theorem, we need to show that there exists a numerical polynomial $\phi\left(t_{1}, \ldots, t_{p}\right)$ in $p$ variables $t_{1}, \ldots, t_{p}$ such that $\phi\left(r_{1}, \ldots, r_{p}\right)=$ Card $U_{r_{1} \cdots r_{p}}^{(2)}$ for all sufficiently large $\left(r_{1}, \ldots, r_{p}\right) \in \mathbb{Z}^{p}$ and $\operatorname{deg}_{t_{i}} \phi \leq m_{i}(i=1, \ldots, p)$.

Let $\operatorname{ord}_{i} u_{A_{j}}^{(1)}=a_{i j}$ and $\operatorname{ord}_{i} u_{A_{j}}^{(i)}=b_{i j}$ for $i=1, \ldots, p ; j=1, \ldots, d$ (clearly, $a_{i j} \leq b_{i j}$ and $a_{1 j}=b_{1 j}$ for $\left.i=1, \ldots, p ; j=1, \ldots, d\right)$. Furthermore, for any $q=1, \ldots, p$ and for any integers $k_{1}, \ldots, k_{q}$ such that $1 \leq k_{1}<\cdots<k_{q} \leq p$, let $V_{j ; k_{1}, \ldots, k_{q}}\left(r_{1}, \ldots, r_{p}\right)=\left\{\theta u_{A_{j}}^{(1)}\right.$ | $\operatorname{ord}_{i} \theta \leq r_{i}-a_{i j}$ for $i=1, \ldots, p$ and $\operatorname{ord}_{k} \theta>r_{k}-b_{k j}$ if and only if $k$ is equal to one of the numbers $\left.k_{1}, \ldots, k_{q}\right\}$. Then $\operatorname{Card} V_{j ; k_{1}, \ldots, k_{q}}\left(r_{1}, \ldots, r_{p}\right)=\phi_{j ; k_{1}, \ldots, k_{q}}\left(r_{1}, \ldots, r_{p}\right)$, where $\phi_{j ; k_{1}, \ldots, k_{q}}\left(t_{1}, \ldots, t_{p}\right)$ is a numerical polynomial in $p$ variables $t_{1}, \ldots, t_{p}$ defined by the formula

$$
\begin{aligned}
\phi_{j ; k_{1}, \ldots, k_{q}}\left(t_{1}, \ldots, t_{p}\right)= & \left(\begin{array}{c}
t_{1}+m_{1}-a_{1 j} \\
m_{1}
\end{array}\right) \ldots\left(\begin{array}{c}
t_{k_{1}-1}+m_{k_{1}-1}-a_{k_{1}-1, j} \\
m_{k_{1}-1}
\end{array}\right) \\
& \times\left[\left(\begin{array}{c}
t_{k_{1}}+m_{k_{1}}-a_{k_{1}, j} \\
m_{k_{1}}
\end{array}\right)-\left(\begin{array}{c}
t_{k_{1}}+m_{k_{1}}-b_{k_{1}, j} \\
m_{k_{1}}
\end{array}\right)\right] \\
& \times\left(\begin{array}{c}
t_{k_{1}+1}+m_{k_{1}+1}-a_{k_{1}+1, j} \\
m_{k_{1}+1}
\end{array}\right) \ldots\left(\begin{array}{c}
t_{k_{q}-1}+m_{k_{q}-1}-a_{k_{q}-1, j} \\
m_{k_{q}-1}
\end{array}\right) \\
& \times\left[\left(\begin{array}{c}
t_{k_{q}}+m_{k_{q}}-a_{k_{q}, j} \\
m_{k_{q}}
\end{array}\right)-\left(\begin{array}{c}
t_{k_{q}}+m_{k_{q}}-b_{k_{q}, j} \\
m_{k_{q}}
\end{array}\right)\right] \\
& \times \cdots\left(\begin{array}{c}
t_{p}+m_{p}-a_{p j} \\
m_{p}
\end{array}\right) .
\end{aligned}
$$

(By Theorem 3.2(iii), Card $\left\{\theta \in \Theta \mid \operatorname{ord}_{1} \theta \leq s_{1}, \ldots, \operatorname{ord}_{p} \theta \leq s_{p}\right\}=\prod_{i=1}^{p}\left(\begin{array}{c}s_{i}+m_{i} \\ m_{i}\end{array}\right)$ for any $s_{1}, \ldots, s_{p} \in \mathbb{N}$.) Clearly, $\operatorname{deg}_{t_{i}} \phi_{j ; k_{1}, \ldots, k_{q}} \leq m_{i}$ for $i=1, \ldots, p$.

Now, for any $j=1, \ldots, d$, let $V_{j}\left(r_{1}, \ldots, r_{p}\right)=\left\{\theta u_{A_{j}}^{(1)} \mid \operatorname{ord}_{i} \theta \leq r_{i}-a_{i j}\right.$ for $i=1, \ldots, p$ and there exists $k \in \mathbb{N}, 1 \leq k \leq p$, such that $\left.\operatorname{ord}_{k} \theta>r_{k}-b_{k j}\right\}$. Applying the combinatorial principle of inclusion and exclusion we obtain that $\operatorname{Card} V_{j}\left(r_{1}, \ldots, r_{p}\right)=$ 
$\phi_{j}\left(r_{1}, \ldots, r_{p}\right)$, where

$$
\begin{aligned}
\phi_{j}\left(t_{1}, \ldots, t_{p}\right)= & \sum_{k_{1}=1}^{p} \phi_{j ; k_{1}}\left(t_{1}, \ldots, t_{p}\right)-\sum_{1 \leq k_{1}<k_{2} \leq p} \phi_{j ; k_{1}, k_{2}}\left(t_{1}, \ldots, t_{p}\right)+\cdots \\
& +(-1)^{v-1} \sum_{1 \leq k_{1}<\cdots k_{v} \leq p} \phi_{j ; k_{1}, \ldots, k_{v}}\left(t_{1}, \ldots, t_{p}\right)+\cdots \\
& +(-1)^{p-1} \phi_{j ; 1,2, \ldots, p}\left(t_{1}, \ldots, t_{p}\right)
\end{aligned}
$$

and Card $U_{r_{1} \cdots r_{p}}^{(2)}$ is an alternating sum of the terms Card $\bigcap_{v=1}^{s} V_{j_{v}}\left(r_{1}, \ldots, r_{p}\right)(1 \leq s \leq$ $\left.d, 1 \leq j_{1}<\cdots<j_{s} \leq d\right)$. Thus, it is sufficient to prove that $\operatorname{Card} \cap_{v=1}^{s} V_{j_{v}}\left(r_{1}, \ldots\right.$, $\left.r_{p}\right)=\phi_{j_{1}, \ldots, j_{s}}\left(r_{1}, \ldots, r_{p}\right)$, where $\phi_{j_{1}, \ldots, j_{s}}\left(t_{1}, \ldots, t_{p}\right)$ is a numerical polynomial such that $\operatorname{deg}_{t_{i}} \phi_{j_{1}, \ldots, j_{s}} \leq m_{i}$ for $i=1, \ldots, p$. If $\bigcap_{v=1}^{s} V_{j_{v}}\left(r_{1}, \ldots, r_{p}\right) \neq \varnothing$ (so that $u_{A_{j_{1}}}^{(1)}, \ldots, u_{A_{j_{s}}}^{(1)}$ contain the same $\Delta$-indeterminate $\left.y_{i}\right)$, then let $v\left(j_{1}, \ldots, j_{s}\right)=\operatorname{lcm}\left(u_{A_{j_{1}}}^{(1)}, \ldots, u_{A_{j_{s}}}^{(1)}\right)$ and let the elements $\gamma_{1}, \ldots, \gamma_{s} \in \Theta$ be defined by the conditions $v\left(j_{1}, \ldots, j_{s}\right)=\gamma_{v} u_{A_{j_{v}}}$ $(v=1, \ldots, s)$. In this case, $\bigcap_{v=1}^{s} V_{j_{v}}\left(r_{1}, \ldots, r_{p}\right)=\left\{u=\theta v\left(j_{1}, \ldots, j_{s}\right) \mid \operatorname{ord}_{i} u \leq r_{i}\right.$ for $i=$ $1, \ldots, p$, and for any $l=1, \ldots, s$, there exists at least one index $k \in\{2, \ldots, p\}$ such that $\operatorname{ord}_{k}\left(\theta \gamma_{l} u_{A_{j_{l}}}^{(k)}\right)$

$\left.>r_{k}\right\}$. Setting $c_{j_{1}, \ldots, j_{s}}^{(i)}=\operatorname{ord}_{i} v\left(j_{1}, \ldots, j_{s}\right)(1 \leq i \leq p)$ and applying the principle of inclusion and exclusion once again, we obtain that Card $\bigcap_{v=1}^{s} V_{j_{v}}\left(r_{1}, \ldots, r_{p}\right)$ is an alternating sum of terms of the form $\operatorname{Card} W\left(j_{1}, \ldots, j_{s} ; k_{11}, k_{12}, \ldots, k_{1 q_{1}}, k_{21}, \ldots, k_{s q_{s}} ; r_{1}, \ldots, r_{p}\right)$, where $W\left(j_{1}, \ldots, j_{s} ; k_{11}, k_{12}, \ldots, k_{1 q_{1}}, k_{21}, \ldots, k_{s q_{s}} ; r_{1}, \ldots, r_{p}\right)=\left\{\theta \in \Theta \mid \operatorname{ord}_{i} \theta \leq r_{i}-c_{j_{1}, \ldots, j_{s}}^{(i)}\right.$ for $i=1, \ldots, p$, and for any $l=1, \ldots, s, \operatorname{ord}_{k} \theta>r_{k}-c_{j_{1}, \ldots, j_{s}}^{(k)}+a_{k j_{l}}-b_{k j_{l}}$ if and only if $k$ is equal to one of the numbers $\left.k_{l 1}, \ldots, k_{l q_{l}}\right\}\left(q_{1}, \ldots, q_{s}\right.$ are some positive integers from the set $\{1, \ldots, p\}$ and $\left\{k_{i v} \mid 1 \leq i \leq s, 1 \leq v \leq q_{s}\right\}$ is a family of integers such that $2 \leq k_{i 1}<k_{i 2}<\cdots<k_{i q_{i}} \leq p$ for $\left.i=1, \ldots, s\right)$.

Thus, it is sufficient to show that $\operatorname{Card} W\left(j_{1}, \ldots, j_{s} ; k_{11}, \ldots, k_{s q_{s}} ; r_{1}, \ldots, r_{p}\right)=$ $\psi_{k_{11}, \ldots, k_{s q_{s}}}^{j_{1}, \ldots, j_{s}}\left(r_{1}, \ldots, r_{p}\right)$ where $\psi_{k_{11}, \ldots, k_{s q_{s}}}^{j_{1}, \ldots, j_{s}}\left(t_{1}, \ldots, t_{p}\right)$ is a numerical polynomial in $p$ variables $t_{1}, \ldots, t_{p}$ such that $\operatorname{deg}_{i} \psi_{k_{11}, \ldots, k_{s} q_{s}}^{j_{1}, \ldots, j_{s}} \leq m_{i}(i=1, \ldots, p)$. But this is almost evident: as in the process of evaluation of Card $V_{j ; k_{1}, \ldots, k_{q}}\left(r_{1}, \ldots, r_{p}\right)$ (when we used Theorem 3.2(iii) to obtain formula (5.3)), we see that Card $W\left(j_{1}, \ldots, j_{s} ; k_{11}, \ldots, k_{s q_{s}} ; r_{1}, \ldots, r_{p}\right)$ is a product of terms of the form $\left(\begin{array}{c}r_{k}+m_{k}-c_{j_{1}, \ldots, j s}^{(k)}-S_{k} \\ m_{k}\end{array}\right)$ (such a term corresponds to an integer $k$ such that $1 \leq k \leq p$ and $k \neq k_{i v}$ for any $i=1, \ldots, s, v=1, \ldots, q_{s}$; the number $S_{k}$ is defined as

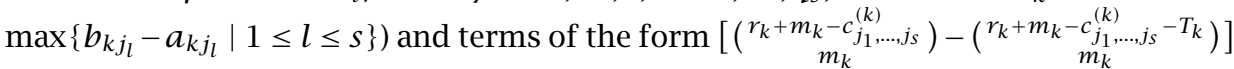
(such a term appears in the product if $k$ is equal to some $k_{i v}\left(1 \leq i \leq s, 1 \leq v \leq q_{s}\right)$. In this case, if $k_{i_{1} v_{1}}, \ldots, k_{i_{e} v_{e}}$ are all elements of the set $\left\{k_{i v} \mid 1 \leq i \leq s, 1 \leq v \leq q_{s}\right\}$ that are equal to $k\left(1 \leq e \leq s, 1 \leq i_{1}<\cdots<i_{e} \leq s\right)$, then $T_{k}$ is defined as $\min \left\{b_{k j_{i_{\lambda}}}-a_{k j_{i_{\lambda}}} \mid\right.$ $1 \leq \lambda \leq l\}$.

The appropriate numerical polynomial $\psi_{k_{11}, \ldots, k_{s q s}}^{j_{1}, \ldots, j_{s}}\left(t_{1}, \ldots, t_{p}\right)$ is a product of $p$ "elementary" numerical polynomials $f_{1}, \ldots, f_{p}$ where $f_{k}(1 \leq k \leq p)$ is either a polynomial of the form $\left(\begin{array}{c}t_{k}+m_{k}-c_{j_{1}, \ldots, j_{s}}^{(k)}-S_{k} \\ m_{k}\end{array}\right)$ or apolynomial of the form $\left[\left(\begin{array}{c}t_{k}+m_{k}-c_{j_{1}, \ldots, j_{s}}^{(k)} \\ m_{k}\end{array}\right)-\right.$ $\left.\left(\begin{array}{c}t_{k}+m_{k}-c_{j_{1}}^{(k)}, \ldots, j_{s} \\ m_{k}\end{array} T_{k}\right)\right]$. Since the degree of such a product with respect to any variable $t_{i}$ $(1 \leq i \leq p)$ does not exceed $m_{i}$, this completes the proof of the theorem. 
DEFINITION 5.2. Numerical polynomial $\Phi_{\eta}\left(t_{1}, \ldots, t_{p}\right)$, whose existence is established by Theorem 5.1, is called a dimension polynomial of the differential field extension $G=K\left\langle\eta_{1}, \ldots, \eta_{n}\right\rangle$ associated with the given system of $\Delta$-generators $\eta=\left\{\eta_{1}, \ldots\right.$, $\left.\eta_{n}\right\}$ and with the given partition of the basic set of derivation operators $\Delta$ into $p$ disjoint subsets $\Delta_{1}, \ldots, \Delta_{p}$.

In what follows, we will consider different lexicographic orders on a set $\mathbb{N}^{p}$ ( $p$ is a positive integer). For any permutation $\left(j_{1}, \ldots, j_{p}\right)$ of the set $\{1, \ldots, p\}$, we define the corresponding lexicographic order $\leq_{j_{1}, \ldots, j_{p}}$ on $\mathbb{N}^{p}$ as follows: $\left(r_{1}, \ldots, r_{p}\right) \leq_{j_{1}, \ldots, j_{p}}\left(s_{1}, \ldots, s_{p}\right)$ if and only if either $r_{j_{1}}<s_{j_{1}}$ or there exists $k \in \mathbb{N}, 1 \leq k \leq p-1$ such that $r_{j_{v}}=s_{j_{v}}$ for $v=1, \ldots, k$ and $r_{j_{k+1}}<s_{j_{k+1}}$. If $\Sigma$ is a finite subset of $\mathbb{N}^{p}$, then an element $e \in \Sigma$ will be called a lex-maximal element of this set, if $e$ is the maximal element of $\Sigma$ with respect to one of the $p$ ! lexicographic orders $\leq_{j_{1}, \ldots, j_{p}}$ (where $\left(j_{1}, \ldots, j_{p}\right)$ is a permutation of the set $\{1, \ldots, p\})$. The set of all lex-maximal elements of a finite set $\Sigma \subseteq \mathbb{N}^{p}$ will be denoted by $\Sigma^{\prime}$. For example, if $\Sigma=\{(3,0,2),(2,1,1),(0,1,4),(1,0,3),(1,1,6),(3,1,0),(1,2,0)\}$ $\subseteq \mathbb{N}^{3}$, then $\Sigma^{\prime}=\{(3,0,2),(3,1,0),(1,1,6),(1,2,0)\}$.

THEOREM 5.3. Let $K$ be a differential field whose basic set $\Delta$ is a union of $p$ disjoint finite sets: $\Delta=\Delta_{1} \cup \cdots \cup \Delta_{p}(p \geq 1)$. Let $G$ be a $\Delta$-field extension of $K$ with the finite set of $\Delta$-generators $\eta=\left\{\eta_{1}, \ldots, \eta_{n}\right\}$ and let

$$
\Phi_{\eta}\left(t_{1}, \ldots, t_{p}\right)=\sum_{i_{1}=0}^{m_{1}} \ldots \sum_{i_{p}=0}^{m_{p}} a_{i_{1} \cdots i_{p}}\left(\begin{array}{c}
t_{1}+i_{1} \\
i_{1}
\end{array}\right) \cdots\left(\begin{array}{c}
t_{p}+i_{p} \\
i_{p}
\end{array}\right)
$$

be the corresponding dimension polynomial $\left(m_{i}=\operatorname{Card} \Delta_{i}\right.$ for $\left.i=1, \ldots, p\right)$. Furthermore, let

$$
E_{\eta}=\left\{\left(i_{1} \cdots i_{p}\right) \in \mathbb{N}^{p}\left(m_{1}, \ldots, m_{p}\right) \mid a_{i_{1} \cdots i_{p}} \neq 0\right\} .
$$

Then the elements $\left(k_{1}, \ldots, k_{p}\right) \in E_{\eta}^{\prime}$ and the corresponding coefficients $a_{k_{1} \cdots k_{p}}$ do not depend on the choice of the system of $\Delta$-generators $\eta$.

Proof. Let $\zeta=\left\{\zeta_{1}, \ldots, \zeta_{q}\right\}$ be another system of $\Delta$-generators of $G$ over $K$ and let

$$
\Phi_{\zeta}\left(t_{1}, \ldots, t_{p}\right)=\sum_{i_{1}=0}^{m_{1}} \cdots \sum_{i_{p}=0}^{m_{p}} b_{i_{1}} \cdots i_{p}\left(\begin{array}{c}
t_{1}+i_{1} \\
i_{1}
\end{array}\right) \cdots\left(\begin{array}{c}
t_{p}+i_{p} \\
i_{p}
\end{array}\right)
$$

be the dimension polynomial of our $\Delta$-field extension associated with the system of generators $\zeta$. Then there exist positive integers $s_{1}, \ldots, s_{p}$ such that $\eta_{i} \in$ $K\left(\bigcup_{j=1}^{q} \Theta\left(s_{1}, \ldots, s_{p}\right) \zeta_{j}\right)$ and $\zeta_{k} \in K\left(\bigcup_{j=1}^{n} \Theta\left(s_{1}, \ldots, s_{p}\right) \eta_{j}\right)(1 \leq i \leq n, 1 \leq k \leq q)$, whence $\Phi_{\eta}\left(r_{1}, \ldots, r_{p}\right) \leq \Phi_{\zeta}\left(r_{1}+s_{1}, \ldots, r_{p}+s_{p}\right)$ and $\Phi_{\zeta}\left(r_{1}, \ldots, r_{p}\right) \leq \Phi_{\eta}\left(r_{1}+s_{1}, \ldots, r_{p}+s_{p}\right)$ for all sufficiently large $\left(r_{1}, \ldots, r_{p}\right) \in \mathbb{Z}^{p}$. It follows that for any element $\left(k_{1}, \ldots, k_{p}\right) \in E_{\eta}^{\prime}$, the term $\left(\begin{array}{c}t_{1}+k_{1} \\ k_{1}\end{array}\right) \cdots\left(\begin{array}{c}t_{p}+k_{p} \\ k_{p}\end{array}\right)$ appears in $\Phi_{\eta}\left(t_{1}, \ldots, t_{p}\right)$ and $\Phi_{\zeta}\left(t_{1}, \ldots, t_{p}\right)$ with the same coefficient $a_{k_{1} \cdots k_{p}}$. This completes the proof of the theorem.

The following example shows that a dimension polynomial of a finitely generated differential field extension associated with some partition of the basic set of derivation operators can carry more differential birational invariants of the extension than the classical Kolchin differential dimension polynomial. 
EXAMPLE 5.4. Let $K$ be a differential field with a basic set of derivation operators $\Delta=\left\{\delta_{1}, \delta_{2}\right\}$ and let $L$ be a $\Delta$-field extension of $K$ generated by a single $\Delta$-generator $\eta$ with the defining equation

$$
\delta_{1}^{a} \delta_{2}^{b} \eta+\delta_{2}^{a+b} \eta=0
$$

where $a$ and $b$ are some positive integers. In other words, $L=K\langle\eta\rangle$ is $\Delta$-isomorphic to the quotient field of the factor ring $K\{y\} / P$, where $P$ is the $\Delta$-ideal of the ring of $\Delta$-polynomials $K\{y\}$ generated by the $\Delta$-polynomial $f(y)=\delta_{1}^{a} \delta_{2}^{b} y+\delta_{2}^{a+b} y$. Let $\omega_{\eta / K}(t)$ be the Kolchin differential dimension polynomial of the extension $L / K$ and let $\Phi_{\eta}\left(t_{1}, t_{2}\right)$ be the dimension polynomial of this extension associated with the partition $\Delta=\left\{\delta_{1}\right\} \bigcup\left\{\delta_{2}\right\}$. By Theorem 4.11, $\{f(y)\}$ is a characteristic set of the ideal $P$. Using the notation of the proof of Theorem 5.1, we obtain that $U_{r_{1} r_{2}}^{(1)}=\left\{u \in \Theta Y \mid \operatorname{ord}_{1} u \leq\right.$ $r_{1}$, ord or $_{2} u \leq r_{2}$ and $\left.\delta_{1}^{a} \delta_{2}^{b} y \nmid u\right\}$ (so that Card $U_{r_{1} r_{2}}^{(1)}=\left(r_{1}+1\right)\left(r_{2}+1\right)-\left(r_{1}+1-a\right)\left(r_{2}+\right.$ $\left.1-b)=b r_{1}+a r_{2}+a+b-a b\right)$ and $U_{r_{1} r_{2}}^{(2)}=\left\{u=\theta \delta_{1}^{a} \delta_{2}^{b} y \mid \theta \in \Theta\left(r_{1}-a, r_{2}-b\right) \backslash \Theta\left(r_{1}-\right.\right.$ $\left.\left.a, r_{2}-(a+b)\right)\right\}$ (so that Card $U_{r_{1} r_{2}}^{(2)}=a\left(r_{1}-a+1\right)$ ). Since $\Phi_{\eta}\left(r_{1}, r_{2}\right)=$ Card $U_{r_{1} r_{2}}^{(1)}+$ Card $U_{r_{1} r_{2}}^{(2)}$ for all sufficiently large $\left(r_{1}, r_{2}\right) \in \mathbb{Z}^{2}$, we obtain that

$$
\Phi_{\eta}\left(t_{1}, t_{2}\right)=(a+b) t_{1}+a t_{2}+2 a+b-a b-a^{2} .
$$

The computation of the Kolchin differential dimension polynomial with the help of the formula in [7, Chapter II, Theorem 6(d)] leads to the following result:

$$
\omega_{\eta / K}(t)=\left(\begin{array}{c}
t+2 \\
2
\end{array}\right)-\left(\begin{array}{c}
t+2-(a+b) \\
2
\end{array}\right)=(a+b) t-\frac{(a+b)(a+b-3)}{2} .
$$

Comparing the polynomials $\omega_{\eta / K}(t)$ and $\Phi_{\eta}\left(t_{1}, t_{2}\right)$, we see that the first polynomial carries two differential birational invariants, its degree 1 and the leading coefficient $a+$ $b$, while $\Phi_{\eta}\left(t_{1}, t_{2}\right)$ carries three such invariants, its total degree $1, a+b$, and $a$ (therefore, $a$ and $b$ are uniquely determined by the polynomial $\left.\Phi_{\eta}\right)$. Thus, $\Phi_{\eta}\left(t_{1}, t_{2}\right)$ gives both parameters $a$ and $b$ of (5.8) while $\omega_{\eta / K}(t)$ gives just the sum of the parameters.

\section{REFERENCES}

[1] B. Buchberger, Ein Algorithmus zum Auffinden der Basiselemente des Restklassenringes nach einem nulldimensionalen Polynomideal, Ph.D. thesis, University of Innsbruck, Austria, 1965.

[2] A. Einstein, Supplement to Appendix II of "The Meaning of Relativity", 4th ed., Princeton University Press, New Jersey, 1953. MR 15,357e.

[3] C. G. J. Jacobi, Gesammelte Werke, vol. 5, G. Reimer, Berlin, 1890 (German).

[4] J. Johnson, Differential dimension polynomials and a fundamental theorem on differential modules, Amer. J. Math. 91 (1969), 239-248. MR 39\#186. Zbl 179.34303.

[5] _ Kähler differentials and differential algebra in arbitrary characteristic, Trans. Amer. Math. Soc. 192 (1974), 201-208. MR 49\#263. Zbl 288.12104.

[6] E. R. Kolchin, The notion of dimension in the theory of algebraic differential equations, Bull. Amer. Math. Soc. 70 (1964), 570-573. MR 29\#5816. Zbl 144.03702.

[7] __ Differential Algebra and Algebraic Groups, Pure and Applied Mathematics, vol. 54, Academic Press, New York, 1973. MR 58\#27929. Zbl 264.12102.

[8] M. V. Kondrat'eva, A. B. Levin, A. V. Mikhalëv, and E. V. Pankrat'ev, Computation of dimension polynomials, Internat. J. Algebra Comput. 2 (1992), no. 2, 117-137. MR 93k\#12012. Zbl 774.12007. 
[9] _ Differential and Difference Dimension Polynomials, Mathematics and its Applications, vol. 461, Kluwer Academic Publishers, Dordrecht, 1999. MR 2001c:12006. Zbl 930.12005.

[10] A. B. Levin, Characteristic polynomials of inverse differential modules and some properties of inverse differential dimension, Russian Math. Surveys 35 (1980), no. 1, 217-218. Zbl 459.12017.

[11] A. B. Levin and A. V. Mikhalëv, A differential dimension polynomial and the rigidity of a system of differential equations, Computable Invariants in the Theory of Algebraic Systems, Akad. Nauk SSSR Sibirsk. Otdel. Vychisl. Tsentr, Novosibirsk, 1987, pp. 58-66 (Russian). MR 90b\#12001. Zbl 722.12004.

[12] A. V. Mikhalëv and E. V. Pankrat'ev, Differential dimension polynomial of a system of differential equations, Algebra, Collection of Papers, Moscow State Univ. Press, Moscow, 1980, pp. 57-67 (Russian). Zbl 0498.12027.

[13] W. Sit, Well-ordering of certain numerical polynomials, Trans. Amer. Math. Soc. 212 (1975), 37-45. MR 53\#10776. Zbl 295.12103.

[14]_ Differential dimension polynomials of finitely generated extensions, Proc. Amer. Math. Soc. 68 (1978), no. 3, 251-257. MR 81f\#12017. Zbl 391.12012.

[15] W. Sit and J. Johnson, On the differential transcendence polynomials of finitely generated differential field extensions, Amer. J. Math. 101 (1979), no. 6, 1249-1263. MR 80m:12029. Zbl 434.12011.

Alexander B. Levin: Department of Mathematics, The Catholic University of AMERICA, WASHINGTON, DC 20064, USA

E-mail address: 1evin@cua.edu 


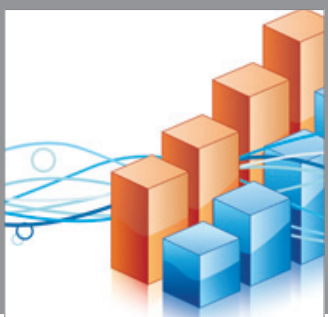

Advances in

Operations Research

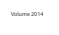

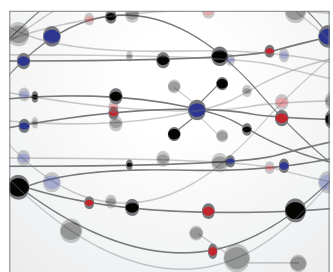

\section{The Scientific} World Journal
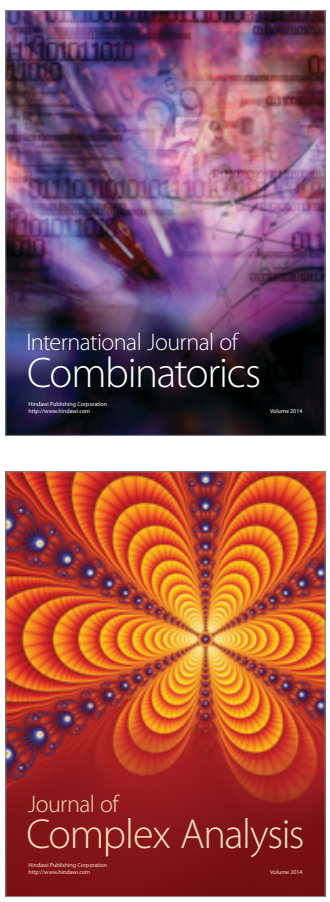

International Journal of

Mathematics and

Mathematical

Sciences
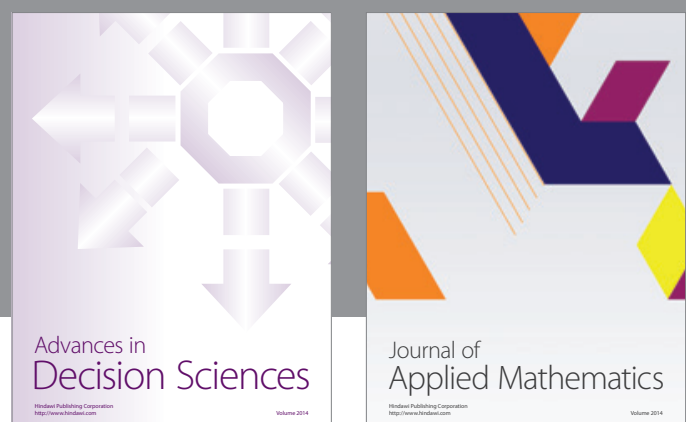

Journal of

Applied Mathematics
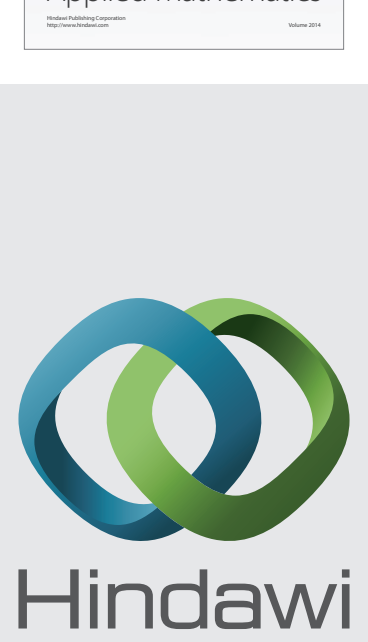

Submit your manuscripts at http://www.hindawi.com
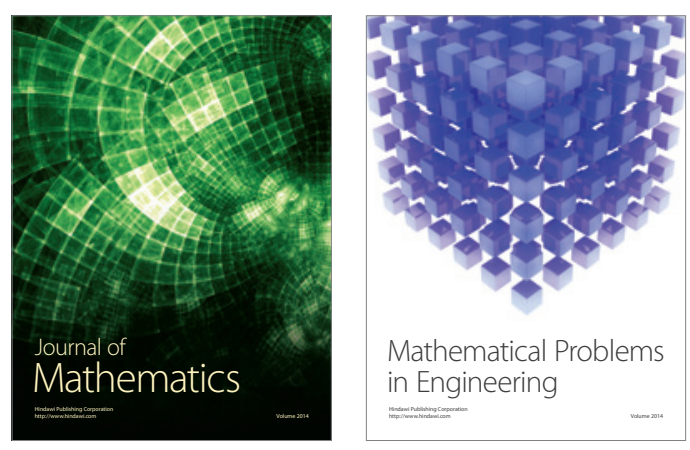

Mathematical Problems in Engineering
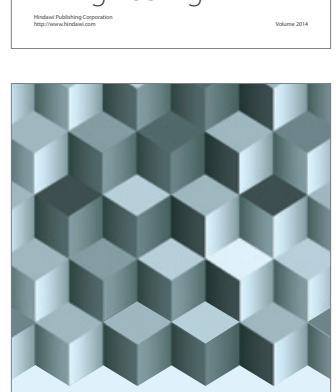

Journal of

Function Spaces
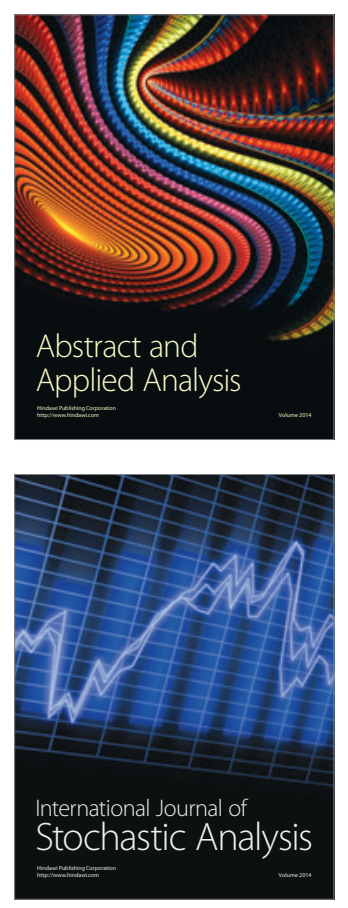

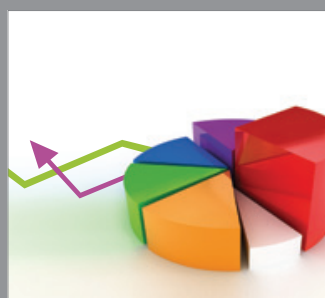

ournal of

Probability and Statistics

Promensencen
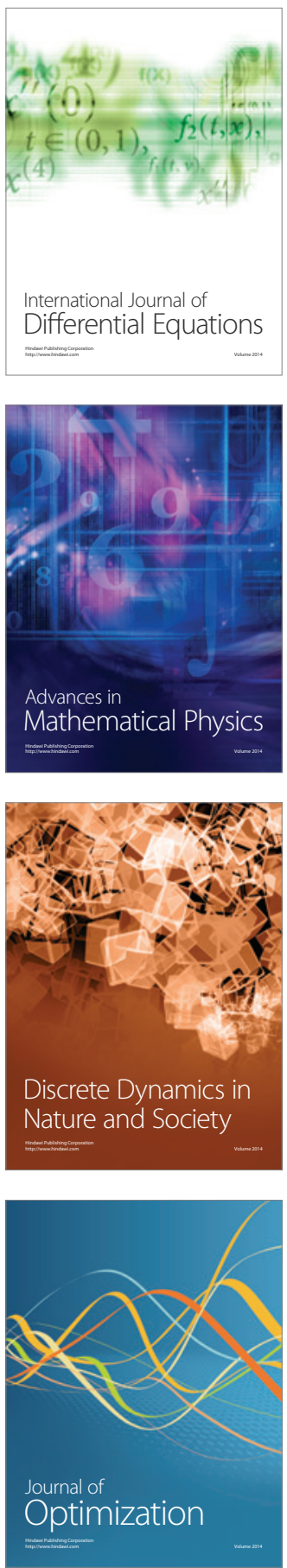